\title{
Periodic Solutions of a System of Delay Differential Equations for a Small Delay
}

\author{
Adu A.M. Wasike and Wandera Ogana
}

Department of Mathematics, University of Nairobi, P.O.Box 30197, Nairobi Kenya, Email: aduwasike@yahoo.com.

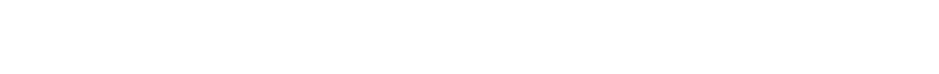

\section{أنو أم. ولسيك و ولندارا لُقلنا}

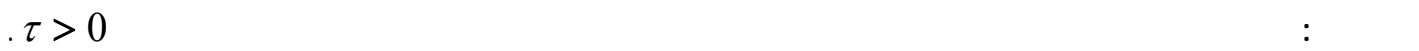

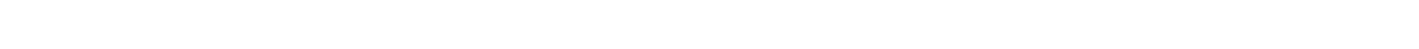

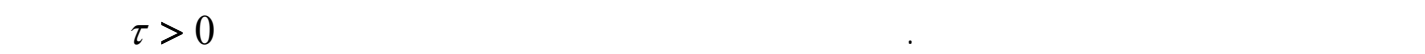

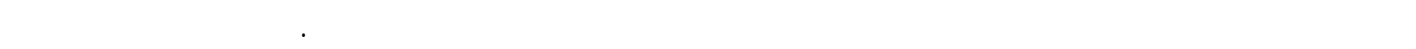
وجود حلول دورية للمعادلات الأصلية عند لستخدلم ظظرية النقة الثابتة لشودرد.

\begin{abstract}
We prove the existence of an asymptotically stable periodic solution of a system of delay differential equations with a small time delay $\tau>0$. To achieve this, we transform the system of equations into a system of perturbed ordinary differential equations and then use perturbation results to show the existence of an asymptotically stable periodic solution. This approach is contingent on the fact that the system of equations with $\tau=0$ has a stable limit cycle. We also provide a comparative study of the solutions of the original system and the perturbed system. This comparison lays the ground for proving the existence of periodic solutions of the original system by Schauder's fixed point theorem.
\end{abstract}

KEYWORDS: Periodic Solutions, Delay Differential Equations, Schauder's Fixed Point Theorem.

\section{Introduction}

1though substantial progress has been made toward the study of first order scalar delay differential equation (DDEs), much less is known about the periodic solutions of systems of DDEs. The need for studying a system of DDEs, aside from its mathematical interest, arises from the wide variety of systems in biology and physical sciences that lead to mathematical models involving such systems. Examples arise in the study of coupled oscillators with a time lag in the coupling (Wasike, 1997), in the diffusion of mass across a membrane, (Epstein, 1990) and in synaptic coupling (Ermentrout,1990).

We consider the system of equations

$$
x^{\prime}(t)=d L[x(t-\tau)-x(t)]+f(x(t))
$$

Where $x(t)=\left[x_{1}(t), x_{2}(t)\right]^{T}$, Here the accent " $"$ " denotes differentiation with respect to time $t$, the superscript $T$ denotes transpose,

$$
L=\left(\begin{array}{ll}
1 & 1 \\
1 & 1
\end{array}\right), \tau, d>0,
$$


$\tau, d>0$, are real numbers, and $f \in C\left(R^{2}\right)$ with $f_{1}(x(t))$ and $f_{2}(x(t))$ are given by

$$
f_{1}\left(x_{1}, x_{2}\right)=-x+x_{1}\left(1-x_{1}^{2}-x_{2}^{2}\right), f_{2}\left(x_{1}, x_{2}\right)=x_{1}+x_{2}\left(1-x_{1}^{2}-x_{2}^{2}\right)
$$

When $|x(t)| \rightarrow 1$, the following hypotheses are satisfied:

$$
\begin{aligned}
& (H 1) x_{2} f_{1}\left(x_{1}, x_{2}\right)<0, \text { for } x_{2} \neq 0 ; x f\left(x_{1}, x_{2}\right)>0 \text {, for } x \neq 0 . \\
& (H 1) \text { Thereis } M>0 \text { such that }\|f(x)\|<M
\end{aligned}
$$

\subsection{Remarks on Hypotheses}

(H1) is a planar negative feedback condition. It forces the solution of equation (1) to rotate about the origin in the anticlockwise sense. $(H 1)$ can be shown to hold for equation (1) by observing that $f_{1}\left(x_{1}, x_{2}\right) \rightarrow-x_{2}$ and $f_{2}\left(x_{1}, x_{2}\right) \rightarrow x_{1}$ as $|x(t)| \rightarrow 1$. The Taylor expansion of $f(x(t))$ about the origin yields $\|f(x(t))\| \leq\|D f(0)\||x(t)| \leq 2=: M$ where $D$ denotes the derivative with respect to $x$.

Equation (1) arises from the study of a model equation of two oscillators coupled by a symmetric-diffusion-like path with a time lag. The equation is given below

$$
X_{i}^{\prime}(t)=d L\left(X_{j}(t-\tau)-X_{i}(t)\right)+f\left(X_{i}(t)\right)
$$

where $X_{k}(t)=\left[x_{k}(t), y_{k}(t)\right]^{T}, i, j, k=1,2, i \neq j$ and $f(X(t))$ is given in (2). After an analysis of the linear part of equation (3), two two-dimensional invariant manifolds

$$
\left\{X_{i}(t): X_{1}(t) \pm X_{2}(t)=0, \forall t \geq-\tau\right\}
$$

were observed with one whose dynamics on the manifold

$$
\left\{X_{i}(t): X_{1}(t)-X_{2}(t)=0, \forall t \geq-\tau\right\}
$$

is described by equation (1), (see Wasike, 1997).

Let $C_{r_{0}}=C\left([-\tau, 0], R^{2}\right)$ with $\|\varphi\|=\sup \{|\varphi(s)|: s \in[-\tau, 0]\} \leq r_{0}<\infty$. Equation (1), subject to an initial condition $\varphi \in C_{r_{0}}$, is known to have a unique solution $x(t ; d, \varphi)$ that satisfies it for $t \geq 0$. The solution is defined on the maximal interval of existence $\left[-\tau, t^{+}(d, \varphi)\right) ; t^{+}(d, \varphi) \leq \infty$, and is continuously differentiable on $\left[0, t^{+}(d, \varphi)\right]$. This solution also depends continuously on the initial condition in the following sense: for every $\varphi \in C_{r_{0}}, t \geq 0$, and $\varepsilon>0$, there exists $\delta>0$ such that for all $\varphi \in C_{r_{0}}$ with $\|\varphi-\psi\| \leq \delta,|x(s ; d, \varphi)-x(s ; d, \psi)|<\varepsilon$, for all $s \in[-\tau, t]$. See for example, Hale and Lunel (1993, Chapter 2).

More information on the basic theory on delay differential equations can be found in Hale and Lunel (1993), Bellman and Cooke (1963) or Diekmann et. al. (1995) ). 
PERIODIC SOLUTIONS OF A SYSTEM OF DELAY DIFFERENTIAL EQUATIONS

\section{Main Results}

We show how a perturbed system of ordinary differential equations (ODE) is found from equation (1) for small delays and then show that the perturbed system has an asymptotically stable periodic orbit.

Proposition 1. equation (1) with $\tau$ small can be written in the form

$$
x^{\prime}(t)=G(x(t) ; d, \tau)+H(x(t)),
$$

where

$$
G(x(t) ; d, \tau):=[I-\eta(d, \tau) L] f(x(t)),
$$

with

$$
\eta(d, \tau):=\frac{d \tau}{1+2 d \tau}
$$

$I$ is a $2 \times 2$ identity matrix, and $\|H(x(t))\|=\left\|x^{\prime}(t)-G(x(t) ; d, \tau)\right\|=O(\tau)$.

Proof. For $I(\tau):=[3 \tau, T], T>3 \tau$, the solution $x(t)$, with $x(0)=\varphi(0)$, is in $C^{3}(I(\tau))$. Therefore

$$
x(t-\tau) \approx x(t)-\tau x^{\prime}(t)+\frac{\tau^{2}}{2} x^{\prime \prime}(t)+\frac{\tau^{3}}{3 !} x^{\prime \prime \prime}(t)+\ldots . .
$$

Let, for notational convenience, equation (1) be written as

$$
x^{\prime}(t)=d L[x(t-\tau)-x(t)]+f(x(t))=: g(x(t-\tau)) \text {. }
$$

For $\tau$ small we have:

$$
(I+d \tau L) g(x(t-\tau))=f(x(t))+d \tau^{2} L\left(\frac{x^{\prime \prime}(t)}{2}-\frac{\tau x^{\prime \prime \prime}(t)}{3 !}+\ldots .\right) .
$$

Simplifying equation (8) we obtain:

$$
g(x(t-\tau))=G(x(t) ; d, \tau)+H(x(t)),
$$

where

$$
H(x(t))=(I-\eta L)\left(\frac{d \tau^{2}}{2} L x^{\prime \prime}(t)+\ldots . .\right),
$$

and $\eta:=\eta(d, \tau)$. It is observed from equation (8) that

$$
\left.x^{\prime \prime}(t)=(I-\eta L)\left[D f(x(t)) x^{\prime}(t)+\frac{d \tau^{2}}{2} L x^{\prime \prime \prime}(t)\right)+\ldots . .\right]
$$

which when substituted in equation (9) gives

$$
H(x(t))=\frac{d \tau^{2}}{2}(1-2 \eta)^{2} L D f(x(t)) x^{\prime}(t)+\ldots .
$$


Clearly $\|H(x(t))\|=O(\tau)$, where $\|\cdot\|$, here is the usual Euclidean norm.

Next we show that the solutions of equations (1) and (4) remain 'close' for all $t \geq 0$. Indeed this also provides another way of proving existence of periodic solutions of equation (1) by Schauder's fixed point theorem.

Proposition 2. The solution $\bar{x}(t)$ of equation (4) is bounded.

Proof. In polar coordinates, defined by

$$
x_{1}(t)=r(t) \cos \theta(t), x_{2}(t)=r(t) \sin \theta(t),
$$

with $|x(t)|=r(t)=: r_{o}$, equation (1) becomes,

$$
\begin{aligned}
& r^{\prime}(t)=r\left(1-r^{2}\right)-d r(1+\sin 2 \theta)+ \\
& d r(t-\tau)\{\cos (\theta(t-\tau)-\theta(t))+\sin (\theta(t-\tau)+\theta(t))\} \\
& \theta^{\prime}(t)=1-d \cos 2 \theta+\frac{d r(t-\tau)}{r(t)}\{\cos (\theta(t-\tau)+\theta(t))+\sin (\theta(t-\tau)-\theta(t))\} .
\end{aligned}
$$

For small $\tau$,

$$
\theta(t-\tau)=\theta(t)-\tau \theta^{\prime}(t)+\text { h.o.t. }, \quad r(t-\tau)=r(t)-\tau \tau^{\prime}(t)+\text { h.o.t. }
$$

where h.o.t. means higher order terms. Using this in equation (10) we get

$$
\begin{aligned}
& r^{\prime}=r\left(1-r^{2}\right)-d\left\{r+r \sin 2 \theta-\left(r-\tau r^{\prime}\right)\left(\cos \tau \theta^{\prime}+\sin \left(2 \theta-\tau \theta^{\prime}\right)\right)\right\} \\
& \theta^{\prime}=1-d\left\{\cos 2 \theta-\left(1-\frac{\tau r^{\prime}}{r}\right)\left(\cos \left(2 \theta-\tau \theta^{\prime}\right)-\sin \tau \theta^{\prime}\right)\right\}
\end{aligned}
$$

By Maclaurins expansion,

$$
\cos \tau \theta^{\prime}=1-\frac{\tau^{2} \theta^{\prime 2}}{2 !}+\text { h.o.t, } \quad \sin \tau \theta^{\prime}=\tau \theta^{\prime}-\frac{\tau^{3} \theta^{\prime 3}}{3 !}+\text { h.o.t. }
$$

Ignoring terms of order higher than $O(\tau)$ and using (12) in (11) we obtain

$$
\left(I_{2}+B(d, \tau)\right)\left(\begin{array}{l}
r^{\prime} \\
\theta^{\prime}
\end{array}\right)=\left(\begin{array}{c}
r\left(1-r^{2}\right) \\
1
\end{array}\right)+R(r, \theta)
$$

where

$$
B(d, \tau)=\left(\begin{array}{ll}
d \tau(1+\sin 2 \theta) & d \tau r \cos 2 \theta \\
(d \tau \cos 2 \theta) / r & d \tau(1-\sin 2 \theta)
\end{array}\right)
$$

and the matrix $R(r, \theta)$ is such that $\|R(r, \theta)\|=O\left(\tau^{2}\right)$. For $\tau$ small $\|B(d, \tau)\|<1$, where $\|\cdot\|$ denotes a norm of a matrix,

$$
\left(I_{2}+B(d, \tau)\right)^{-1} \approx I_{2}-B(d, \tau) .
$$

Thus ignoring terms of $O\left(\tau^{2}\right)$ in (13), we obtain

$$
r^{\prime}=r\left(1-r^{2}\right)(1-d \tau(1+\sin 2 \theta))-d \tau r \cos 2 \theta
$$


PERIODIC SOLUTIONS OF A SYSTEM OF DELAY DIFFERENTIAL EQUATIONS

$$
\theta^{\prime}=1-d \tau(1-\sin 2 \theta)-d \tau\left(1-r^{2}\right) \cos 2 \theta
$$

Solving equation (14), and noting that for fixed $\theta, \bar{r}:=|\bar{x}(t)|$ is given by

$$
\bar{r}=\left[1-\frac{d \tau \cos 2 \theta}{1-d \tau(1+\sin 2 \theta)}\right]^{\frac{1}{2}}
$$

By Binomial expansion,

$$
\bar{r}=1-\frac{1}{2} \frac{d \tau \cos 2 \theta}{1-d \tau(1+\sin 2 \theta)}+\ldots
$$

Clearly $\bar{r} \approx 1 \pm O(\tau)$ used in equation (14), shows that $\bar{x}(t)$ is bounded.

We now give other comparisons of the results between the solution $\bar{x}(t):=\bar{x}(t ; d, \tau)$ of the perturbed system equation (4) subject to initial data $\bar{x}(\tau):=\left[\bar{x}_{1}(0), 0\right]^{T}$ and the solution $x(t, d, \varphi)$ to equation (1) subject to the initial condition $\varphi(0)=\left[\varphi_{1}(0), 0\right]^{T}$. Let $\bar{x}_{1}(\tau):=\bar{x}_{1}(0)=\varphi_{1}(0)$ and $\bar{T}$ the first time of return of $\bar{x}(t)$ such that $\bar{x}_{1}(\bar{T})>0$ and $\bar{x}_{2}(\bar{T})=0$. We note from the proof of Proposition 2 that $\|x(t)\| \leq r_{0}$ and $\|\bar{x}(t)\| \leq \bar{r}$. Define the set

$$
\mathrm{B}(\bar{x}(\tau)) \stackrel{\Delta}{=}\left\{\varphi \in C_{r 0}:\|\varphi(s)-\bar{x}(\tau)\| \leq O(\tau),-\tau \leq s \leq 0\right\}
$$

with $\|\cdot\|$ denoting the Euclidean norm. The set $\mathrm{B}(\bar{x}(\tau))$ is a closed bounded set. By taking initial values in this set, we wish to show that $\bar{x}(t)$ and $x(t)$ remain $o(\tau)$ 'close'; that is, $\|x(t)-\bar{x}(t)\|=o(\tau)$ for all $t \geq 0$. This is accomplished by using Lemmas 1 and 2 below.

Lemma 1. For any $\varphi \in \mathrm{B}(\bar{x}(\tau)),\|\bar{x}(t)-x(t)\|=o(\tau)$ for all $t \geq 0$.

Proof. Equation (1) can be written as $x^{\prime}=G(x(t) ; d, \tau)+H(x(t) ; d, \tau)$. Let

$$
\begin{aligned}
& \bar{x}^{\prime}(t)=G(x(t) ; d, \tau) . \\
& \frac{d}{d t}[x(t)-\bar{x}(t)]=G(x(t))-G(\bar{x}(t))+H(x(t))
\end{aligned}
$$

where $G(x(t)):=G(x(t) ; d, \varphi), G(\bar{x}(t)):=G(\bar{x}(t) ; d, \tau)$ and $H(x(t)):=H(x(t) ; d, \tau)$. By using the inner product in $R^{2}$, we get

$$
\begin{gathered}
\frac{1}{2} \frac{d}{d t}\|x(t)-\bar{x}(t)\|^{2} \leq\|x(t)-\bar{x}(t)\|\left\{x^{\prime}(t)-\bar{x}^{\prime}(t)\right\}, \\
\|x(t)-\bar{x}(t)\| D_{+}\|x(t)-\bar{x}(t)\| \leq(G(x(t)) G(\bar{x}(t))+H(x(t)))\|x(t)-\bar{x}(t)\|,
\end{gathered}
$$

where $D_{+}$denotes the derivative from the right. This simplifies to 


$$
\|x(t)-\bar{x}(t)\| D_{+}\|x(t)-\bar{x}(t)\| \leq(\|G(x(t))-G(\bar{x}(t))\|+\|H(x(t))\|)\|x(t)-\bar{x}(t)\| .
$$

Since $G \in C^{1}$ on $\mathrm{B}(\bar{x}(\tau))$, it is Lipchtzian with a Lipschitz constant $0<k$ (say). From this we obtain

$$
D_{+}\|x(t)-\bar{x}(t)\| \leq k\|x(t)-\bar{x}(t)\|+\| H(x(t) \|,
$$

Since $\|H(x(t))\|=o(\tau)$, we have $D_{+}\|x(t)-\bar{x}(t)\| \leq k\|x(t)-\bar{x}(t)\|+o(\tau)$

Using the Gronwall's Inequality and bearing in mind that $\varphi(0)=\bar{x}(0)$, we get

$$
\|x(t)-\bar{x}(t)\| \leq o(\tau) \int_{0}^{t} \exp (k(t-s)) d s=o(\tau),
$$

Hence the lemma is proved.

\section{Lemma 2.}

Let $N_{\varepsilon}(\bar{T})$ be an $\varepsilon>0$ neighborhood of $\bar{T}$. Then

(i) There is $T * \in\left[T_{1}, T_{2}\right]$, with $\bar{x}_{2}\left(T_{1}\right)<0, \bar{x}_{2}\left(T_{2}\right)>0, T_{1}, T_{2} \in N_{\varepsilon}(\bar{T})$, such that $x_{2}\left(T^{*} ; d, \varphi\right)=0, x_{1}\left(T^{*} ; d, \varphi\right)>0$, and

(ii) $\left|T^{*}-\bar{T}\right|=O(\tau)$.

Proof (i). We show the existence of $T^{*}$. Without loss of generality, choose $\varepsilon>0$ and small enough such that $T_{1}=\bar{T}-\varepsilon, T_{2}=\bar{T}+\varepsilon$. Then $\bar{x}_{2}\left(T_{1}\right)=\bar{x}_{2}(\bar{T})-\varepsilon x_{2}^{\prime}(\bar{T})+$ h.o $t$. Recall that $\bar{x}_{2}(\bar{T})=0$. By the continuity of $f_{1}(\bar{x}(t))$ and Hypothesis $H 1, f_{1}(\bar{x}(\bar{T})) \rightarrow 0$ as $\bar{x}_{2}(t) \rightarrow 0$, and using $\eta=\eta(d, \tau) \rightarrow 0$ as $\tau \rightarrow 0$, we have

$$
\bar{x}_{2}\left(T_{1}\right)=-\varepsilon(1-\eta) f_{2}(\bar{x}(\bar{T})) \rightarrow-\varepsilon \bar{x}_{1}(\bar{T})=-\varepsilon \bar{r} \neq 0
$$

Similarly $\bar{x}_{2}\left(T_{2}\right)=\varepsilon \bar{r}$. By the fact that, for any $\varphi \in B(\bar{x}(\tau)),\|x(t)-\bar{x}(t)\|=o(\tau)$ uniformly in any bounded set in $t$, we have $\left|x_{2}\left(T_{1}\right)-\bar{x}_{2}\left(T_{1}\right)\right|=o(\tau)$, implying $x_{2}\left(T_{1}\right)=\bar{x}_{2}\left(T_{1}\right)+o(\tau)$. But $\bar{x}_{2}\left(T_{1}\right)=-\varepsilon \bar{r}$. Hence $x_{2}\left(T_{1}\right)=-\varepsilon \bar{r}+o(\tau)$. Similarly $\quad x_{2}\left(T_{2}\right)=-\varepsilon \bar{r}+o(\tau)$. Ignoring $O(\tau)$ and noting that $x(t)$ is at least $C^{l}$ on $\left[T_{1}, T_{2}\right]$, by the intermediate value theorem, then there is a $T^{*}:=T(d, \tau) \in\left[T_{1}, T_{2}\right]$ such that $x_{2}\left(T^{*}\right)=0$.

Proof (ii). We prove that $\left|\bar{T}-T^{*}\right|=O(\tau)$. In the neighborhood of $\bar{T}$, we can use linear interpolation to determine $\bar{T}$ and $T^{*}$. For $\bar{x}_{2}(t), t \in\left[T_{1}, T_{2}\right]$ we have

$$
\bar{T}=\frac{T_{1} \bar{x}_{2}\left(T_{2}\right)-T_{2} \bar{x}_{2}\left(T_{1}\right)}{\bar{x}_{2}\left(T_{2}\right)-\bar{x}_{2}\left(T_{1}\right)}=\frac{T_{1}+T_{2}}{2}
$$




\section{PERIODIC SOLUTIONS OF A SYSTEM OF DELAY DIFFERENTIAL EQUATIONS}

and for $x_{2}(t), t \in\left[T_{1}, T_{2}\right]$

$$
T^{*}=\frac{T_{1} x_{2}\left(T_{2}\right)-T_{2} x_{2}\left(T_{1}\right)}{x_{2}\left(T_{2}\right)-x_{2}\left(T_{1}\right)}=\frac{\left(T_{1}+T_{2}\right) \varepsilon \bar{r}+\left(T_{1}-T_{2}\right) o(\tau)}{2 \varepsilon \bar{r}}
$$

Therefore $\quad \bar{T}-T^{*}=-\frac{\left(T_{1}-T_{2}\right) o(\tau)}{2 \varepsilon \bar{r}}$;

and by $T_{1}-T_{2}=-2 \varepsilon, \bar{T}-T^{*}=o(\tau) / r$. Recall that $\bar{r}=1+o(\tau)$. Thus $\left|\bar{T}-T^{*}\right|=o(\tau)$.

We now demonstrate that any solution of equation (1) with initial value in $B(\bar{x}(\tau))$ returns to it after some $T^{*}>0$. This is shown by Lemmas 3 and 4 below.

First we show that at the time $\bar{T}>0,\left(T^{*}>0\right)$ of crossing the positive part of the $\bar{x}_{1}(t)$ (respectively, $\left.x_{1}\right)$ axis, the solutions $\bar{x}(t)$, and $(x(t))$ are still $o(\tau)$ close.

Lemma 3. $\left\|\bar{x}(\bar{T})-\bar{x}\left(T^{*}\right)\right\|=o(\tau)$

Proof.

$$
\begin{aligned}
& \left\|\bar{x}(\bar{T})-\bar{x}\left(T^{*}\right)\right\|=\left\|\left(\left(\begin{array}{l}
\bar{x}_{1}(\bar{T})-\bar{x}_{1}(\bar{T}) \\
\bar{x}_{2}(\bar{T})-\bar{x}_{2}(\bar{T})
\end{array}\right)\right)+\left(\bar{T}-T^{*}\right)\left(\begin{array}{l}
\bar{x}_{1}^{\prime}(\bar{T}) \\
\bar{x}_{2}^{\prime}(\bar{T})
\end{array}\right)\right\| \\
& =\left|\bar{T}-T^{*}\right|\left(\bar{x}_{1}^{\prime}(\bar{T})^{2}+\bar{x}_{2}(\bar{T})^{2}\right)^{\frac{1}{2}}, \\
& \bar{x}_{1}^{\prime}(\bar{T})=(1-\eta) f_{1}(\bar{x}(\bar{T}))-\eta f_{2}(\bar{x}(\bar{T})) \text {, } \\
& \bar{x}_{2}^{\prime}(\bar{T})=-\eta f_{1}(\bar{x}(\bar{T}))+(1-\eta) f_{2}(\bar{x}(\bar{T})) \text {. }
\end{aligned}
$$

By the continuity of $f(x(t))$ and Hypothesis (H1), we obtain

$$
\left[\bar{x}_{1}(\bar{T})^{2}+\bar{x}_{2}(\bar{T})^{2}\right]^{\frac{1}{2}}=\bar{x}_{1}(t)\left(1-2 \eta+2 \eta^{2}\right)^{\frac{1}{2}}
$$

which tends to $\bar{x}_{1}(\bar{T})=\bar{r}$ as $\tau \rightarrow 0$. Hence, the expression in (18) above gives $\left\|\bar{x}(\bar{T})-\bar{x}\left(T^{*}\right)\right\|=\left|T^{*}-\bar{T}\right| \bar{r}$ and, by using $\left|T^{*}-\bar{T}\right|=o(\tau)$, we have, $\left\|\bar{x}(\bar{T})-\bar{x}\left(T^{*}\right)\right\|=o(\tau)$.

Lemma 4. For any $t \in\left[T^{*}-\tau, T^{*}\right]$ and $\varphi \in \mathrm{B}(\bar{x}(\bar{T})),\left\|x(t)-x\left(T^{*}\right)\right\|=o(\tau)$

Proof. By the mean value theorem, and Lemma 1,

$$
\begin{aligned}
& \left\|x(t)-x\left(T^{*}\right)\right\| \leq \tau \sup _{t \in\left[T^{*}-\tau, T^{*}\right]}\left\|\frac{d}{d t} x(t)\right\|=\tau \sup _{t \in\left[T^{*}-\tau, T^{*}\right]}\left\{\left\|\frac{d}{d t}(\bar{x}(t)+o(\tau))\right\|\right\} \\
& =\tau \sup _{t \in\left[T^{*}-\tau, T^{*}\right]}\{\|(I-\eta L) f(x(t))\|\} \leq \tau(1+2 \eta) M=o(\tau)
\end{aligned}
$$

Proposition 3. For any $\varphi \in B(\bar{x}(\tau)), x_{T^{*}}=\left.x(t)\right|_{\left[T^{*}-\tau, T^{*}\right]} \in B(\bar{x}(\tau))$. 
Proof. The proof of this proposition shows that indeed $x(t)$ returns to $\mathrm{B}(\bar{x}(\tau))$ after some time $t \in\left[T^{*}-\tau, T^{*}\right]$. We first show that $\left\|x\left(T^{*}\right)-\bar{x}(\tau)\right\| \leq o(\tau)$. We observe that

$$
\left\|\bar{x}(\tau)-x\left(T^{*}\right)\right\| \leq\|\bar{x}(\tau)-\bar{x}(\bar{T})\|+\left\|\bar{x}(\bar{T})-\bar{x}\left(T^{*}\right)\right\|+\left\|\bar{x}\left(T^{*}\right)-x\left(T^{*}\right)\right\| .
$$

Let us consider each term in turn.

$$
\|\bar{x}(\tau)-\bar{x}(\bar{T})\|=\left\|\left(\left(\begin{array}{c}
\bar{x}_{1}(\tau) \\
0
\end{array}\right)-\left(\begin{array}{c}
\bar{x}_{1}(\bar{T}) \\
0
\end{array}\right)\right)\right\| \leq\left\|r_{0}+o(\tau)-r_{0}\right\|=o(\tau)
$$

By Lemma 3, $\left\|\bar{x}(\bar{T})-\bar{x}\left(T^{*}\right)\right\|=O(\tau)$, and by Lemma $1,\left\|\bar{x}\left(T^{*}\right)-x\left(T^{*}\right)\right\|=O(\tau)$. Thus $\left\|\bar{x}(\tau)-x\left(T^{*}\right)\right\|=O(\tau)$.

Let $t \in\left[T^{*}-\tau, T^{*}\right],\|x(t)-\bar{x}(\tau)\| \leq\left\|\bar{x}(\tau)-x\left(T^{*}\right)\right\|+\left\|x\left(T^{*}\right)-x(\tau)\right\|$. By the estimate above and Lemma 4 we see that $\|x(t)-\bar{x}(\tau)\| \leq 2 o(\tau)=o(\tau)$, implying $x_{T^{*}} \in B(\bar{x}(\tau))$. The proof of proposition 4 , shows that indeed $x(t)$ returns to $\mathrm{B}(\bar{x}(\tau))$ at $x_{T^{*}}$.

Theorem 5. Equation (1) with small $\tau$ has at least one periodic solution.

Proof. This follows from Proposition 4 and Lemmas 1-4 above. Indeed we can define the Poincar'e operator $\rho: \varphi \in \mathrm{B}(\bar{x}(t)) \rightarrow x_{T^{*}} \in C_{r o}$. By Proposition 4, $\rho$ is defined from a closed bounded convex set $\mathrm{B}(\bar{x}(t))$ into itself. The continuity of $\rho$ is assured by the continuous dependence of solutions on the initial data (as shown in Lemma 1) and the transversality of $x(t)$ with the $x_{1}$-axis (as essentially proved in Lemma $2(i)$ ). Since $\rho$ maps $B$, a compact set, into itself, and is continuous, it is completely continuous. By Schauder's fixed-point Theorem we conclude that $\rho$ has at least one fixed point, which corresponds to a periodic solution of equation (4).

\section{References}

BELLMAN, R., and COOKE,K., 1963. Differential-Difference Equations. Academic Press, New York.

DIEKMANN,O.G., S.A., LUNEL S.M.V., WALTER, H.-O., 1995. Delay Equations SpringerVerlag. New-york, Berlin, Heidelberg.

HALE. J.K., and LUNEL, S.M.V. 1993. Introduction to Functional Differential Equations. Springer-Verlag. New-york, Berlin, Heidelberg

EPSTEIN, I.R. 1990. Differential delay equations in chemical kinematics: Some simple linear model systems. J. Chem. Phys. 92: 1702-1713.

ERMENTROUT, G.B., and KOPPELL, N. 1990. Oscillator death in a system of coupled Nueral oscillator. SIAM J. Appl. Maths. 50: 125-146.

HALE, J.K. 1980. Ordinary Differential Equations (2nd edition) Krieger, Malabar.

WASIKE, A.A. M. 1997. An Analytical and Numerical Study of A System of Coupled Oscillators With a Time Lag in the Coupling. University of Nairobi. Ph.D. Thesis University of Nairobi. 